\title{
Continuous Regional Analysis Device for Neonate Lung (CRADL)
}

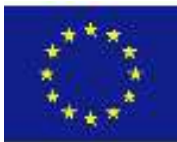

Richard Bayford, Andrew Tizzard, Inéz Frerichs, Norbert Weiler, Christina Karaoli, Stelios Christofides, Charalambos Yiannakkaras, Peter Rimensberger, Sven Nordebo, Stephan H. Böhm, Anton van Kaam, Bruce Fifield, Christopher Knox, Lotte Steuten, Gimon de Graaf, Rebecca Yerworth, Micheal Butterworth, and Andreas Demosthenous.

correspondance address: r.bayford@mdx.ac.uk

Abstract - Each year 15 million babies are born prematurely and many suffer from respiratory failure due to immaturity of the lung and lack of control of breathing. Although respiratory support, especially mechanical ventilation, can improve their survival, it may also cause injury to the vulnerable lung resulting in severe and chronic pulmonary morbidity, lasting into adulthood. Heterogeneity of lung aeration, with areas of over inflation and collapse, plays a crucial part in the mortality and morbidity due to respiratory failure. This regionally different distribution of lung aeration cannot be detected by currently available bedside monitoring tools and imaging methods. Thus, an imaging technique for continuous non-invasive bedside monitoring of infants' lung function is urgently needed. In order to address this, CRADL will use EIT technology to establish a tool for monitoring regional lung function and to guide interventions in the paediatric population.

Electrical impedance tomography (EIT) is a non-radiative, inexpensive technique that can facilitate real time monitoring of lung aeration, and recent studies have shown that it is effective in monitoring aeration in preterm babies. CRADL will show how EIT can provide new cost effective, easy to use, respiratory management tools and clinical protocols that can be universally adopted to reduce deaths and disability in preterm babies by delivering a clinical device that provides continuous, non-invasive, radiation free, bedside information on regional lung aeration and ventilation during daily clinical care of (preterm) infants and children with respiratory failure. CRADL will also assess the effectiveness, efficacy and safety of such a system in guiding respiratory management and supportive care of the most common causes of paediatric respiratory failure (respiratory distress syndrome, bronchiolitis and acute respiratory distress syndrome), with the final goal of reducing short and long term adverse effects of disease and side effects of its treatment.

This project has received funding from the European Union's Horizon 2020 research and innovation programme under grant agreement No 668259. This reflects only the author's views and that the Commission is not responsible for any use that may be made of the information it contains. 